\title{
Emerging Computational Strategy for Eradication of Malaria
}

\author{
${ }^{1}$ Monday Eze, ${ }^{2}$ Jane Labadin and ${ }^{3}$ Terrin Lim \\ Faculty of Computer Science and Information Technology, \\ Universiti Malaysia Sarawak, 94300 Kota Samarahan, Malaysia \\ 1eze_monday@yahoo.com, ${ }^{2}$ ljane@fit.unimas.my and ${ }^{3}$ terrin@fit.unimas.my
}

\begin{abstract}
Even with a lot of efforts by researchers, malaria eradication is yet to become a reality. The link between malaria infected and non-infected human beings, which led to transmission of the disease, is bridged by infected malaria vectors, through blood sucking bites. Such bites take place either in residential homes or public places. Suppose the residential homes are placed under reliable vector control, malaria transmission will still persist unless the public places are taken into consideration. The emerging computational strategy is about leveraging on the interactions between human beings and mosquitoes in public places to build realistic network models with the ultimate aim of applying relevant search techniques to such models, so as to determine the public places which act as reservoir of infected malaria vectors. The overall result will form a key resource for realistic vector control. Hence the idea is to be more proactive and offensive against malaria vectors, by locating the vectors in their hiding places, rather than human beings being located and bitten by the infected vectors. The emerging computational strategy discussed here is part of an ongoing research project in application of computational techniques towards eradication of malaria. This paper documents the result of an initial survey on malaria vector existence in public places.
\end{abstract}

\section{INTRODUCTION}

A number of public places, such as bars and restaurants have been reported to close outdoor terraces or shut down completely as customers cannot sit outside because of what experts described as 100 billion mosquitoes invasion [1]. The importance of public places in disease transmission could be proved by referring to research by [2] and [3] which shows that contact rate between infectious and susceptible individuals could be reduced as much as $50 \%$ by closing some public places. While closing down public places is not the best solution, this finding however underlines the importance of public places in disease propagation. It has been shown that the number of individuals in a household drastically affects the size of disease outbreaks [2]. This implies that the more the number of people interacting in a place, the more the probability of disease transmission in that location. A recent literature survey carried out as part of this research revealed that the average household size for all the countries studied spans between 2.2 and 7.4. For instance Bangladesh [4] is 5.6, Norway [5] is 2.3, Switzerland [6] is 2.2, Japan [7] is 2.7, Afghanistan [8] is 7.4, Australia [9] is 2.6, China [10] is 3.7, Egypt [11] is 4.9, USA [12] is 2.57 and Canada [12] is 3.1. Since most public places (eg. schools, markets, airports etc) accommodate thousands of people, the probability of disease spread is very high in such places, and hence the need for studying malaria transmission in public places.

\section{RESEARCH HYPOTHESES:}

The hypotheses for this research experiment are:

Hypothesis 1: Some public places where human beings visit for their daily socio-economic activities harbor malaria vectors.

Hypothesis2: These public places contribute to propagation of malaria due to blood sucking bites by mosquitoes in these locations.

Hypothesis3: The control of malaria resulting from human migration from location to location will remain very difficult to deal with, as long as malaria vectors thrive in public places.

Hypothesis4: Network Modeling can be used to study the dynamics of malaria transmission in public places.

Hypothesis5: Even if vectors are controlled effectively in the residential homes, malaria will still persist if the public places are not taken into consideration.

\section{MATERIALS AND METHODS}

A survey research was carried out in Sarawak State of Malaysia in June 2010 on vector existence in public places. A total of 525 valid responses were obtained from respondents drawn from ten countries, though the indigenes (Malaysian citizens) constituted a greater percentage. The foreigners involved were those who visited or were living in Malaysia at the time of this research. The aim of this survey is to determine previous experiences of mosquito bites in 16 carefully selected type of public places (see TABLE I). The respondents also had the option of indicating three most critical public places, and ranking them in the order of perceived degree of vector existence (or mosquito bites experiences), as well as suggesting other public places apart from the sixteen. The vector control strategies used in residential homes were also studied. Various modeling and data analysis techniques were applied to derive relevant outputs. The two popular correlation coefficients (Spearman and Pearson Correlation Coefficients) were applied for relevant validation of the research findings. 